\title{
ARTICLE
}

\section{A paper-based microfluidic biosensor integrating zinc oxide nanowires for electrochemical glucose detection}

\author{
Xiao Li, Chen Zhao and Xinyu Liu
}

This paper reports an electrochemical microfluidic paper-based analytical device (E $\mu \mathrm{PAD})$ for glucose detection, featuring a highly sensitive working electrode (WE) decorated with zinc oxide nanowires (ZnO NWs). In addition to the common features of $\mu$ PADs, such as their low costs, high portability/disposability, and ease of operation, the reported E $\mu$ PAD has three further advantages. (i) It provides higher sensitivity and a lower limit of detection (LOD) than previously reported $\mu$ PADs because of the high surface-to-volume ratio and high enzyme-capturing efficiency of the ZnO NWs. (ii) It does not need any light-sensitive electron mediator (as is usually required in enzymatic glucose sensing), which leads to enhanced biosensing stability. (iii) The ZnO NWs are directly synthesized on the paper substrate via low-temperature hydrothermal growth, representing a simple, low-cost, consistent, and mass-producible process. To achieve superior analytical performance, the on-chip stored enzyme (glucose oxidase) dose and the assay incubation time are tuned. More importantly, the critical design parameters of the E $\mu \mathrm{PAD}$, including the WE area and the ZnO-NW growth level, are adjusted to yield tunable ranges for the assay sensitivity and LOD. The highest sensitivity that we have achieved is $8.24 \mu A \cdot \mathrm{mM}^{-1} \cdot \mathrm{cm}^{-2}, \mathrm{with}$ a corresponding LOD of $59.5 \mu \mathrm{M}$. By choosing the right combination of design parameters, we constructed E $\mu$ PADs that cover the range of clinically relevant glucose concentrations $(0-15 \mathrm{mM})$ and fully calibrated these devices using spiked phosphate-buffered saline and human serum. We believe that the reported approach for integrating ZnO NWs on E $\mu$ PADs could be well utilized in many other designs of $\mathrm{E} \mu \mathrm{PADs}$ and provides a facile and inexpensive paradigm for further enhancing the device performance.

Keywords: electrochemical detection; enzymatic biosensor; glucose detection; paper-based microfluidics; zinc oxide nanowire

Microsystems \& Nanoengineering (2015) 1, 15014; doi:10.1038/micronano.2015.14; Published online: 3 August 2015

\section{INTRODUCTION}

Microfluidic paper-based analytical devices ( $\mu$ PADs) represent a newly emerging platform for applications such as diagnostic biosensing and environmental monitoring ${ }^{1}$. MicroPADs can be fabricated at low cost and used with ease in resource-limited settings by end users without professional skills ${ }^{2}$. These merits make them particularly promising for promoting public health in developing countries, where the costs of diagnoses are a major concern and resources such as electricity and professional equipment are not always accessible. They may also contribute to health care in the developed world because they could eventually enable users to conveniently self-monitor their health conditions in a point-of-care fashion. Many $\mu P A D$ designs adopt electrochemical sensing mechanisms (which are thus called electrochemical microfluidic paper-based analytical devices or E $\mu$ PADs) because of the quantitative nature, high accuracy and high sensitivity of these mechanisms ${ }^{3-5}$.

With rapid advances in the field of paper-based microfluidics, research efforts are shifting focus to the creation of fully functional $\mu \mathrm{PAD} s$ with superior analytical performance for practical uses ${ }^{6}$, for which the introduction of biosensing nanomaterials to $\mu$ PADs has shown remarkable potential. The benefits of using nanomaterials for biosensing primarily stem from their unique physical/chemical characteristics ${ }^{7}$. For instance, researchers have integrated multi-walled carbon nanotubes (MWCNTs) into $\mu$ PADs to covalently immobilize probe antibodies and enhance the signal output of electrochemical immunoassays by utilizing MWCNT's high surface-to-volume ratio and superior electrical conductivity ${ }^{4}$. Graphene and gold nanorods have also been utilized on $\mu$ PADs for electrochemical ${ }^{8}$ and optical biosensing ${ }^{9}$, respectively. Nevertheless, the preparation of these nanomaterials could be complex and costly, which may hinder the wide use of $\mu$ PADs. In addition, the integration of these nanomaterials into the $\mu$ PADs after synthesis may also raise concerns about the stability of the nanomaterials' attachment to and the uniformity of their distribution on the paper substrates.

Zinc oxide nanowires ( $\mathrm{ZnO} \mathrm{NWs}$ ) have shown outstanding capacities in biosensing ${ }^{10}$. They possess not only high surface-tovolume ratios but also high biocompatibility and fast electron transfer. Additionally, ZnO NWs have an isoelectric point (IEP) of approximately 9.5, which is much higher than that of many enzymes and therefore allows the $\mathrm{ZnO} N W s$ to immobilize enzymes with high efficiency via electrostatic attraction. As a result, $\mathrm{ZnO} \mathrm{NWs}$ have been a popular option for enzymatic electrochemical sensing of targets such as glucose ${ }^{11}$, uric acid ${ }^{12}$, and phenol ${ }^{13}$. Another highly desirable property of $\mathrm{ZnO} N W s$ is that they can be synthesized on various substrates (e.g., plastics ${ }^{14}$ and cellulose paper ${ }^{15}$ ) through a low-cost hydrothermal process. To date, there is no previous work on the development of $\mu$ PADs that use $\mathrm{ZnO}$ nanomaterials as the biosensing component.

Here, we report the first E $\mathrm{PAD}$ with $\mathrm{ZnO}$ NWs synthesized in situ for electrochemical enzymatic detection of glucose in human serum. An array of circular paper channels (reaction zones) are formed on a paper substrate by wax patterning, and electrodes are patterned on top of the reaction areas by stencil printing of conductive inks. The E $\mu$ PAD features a separate layer of working electrode (WE), which is formed by covering a paper piece with carbon ink and then growing $\mathrm{ZnO} \mathrm{NWs}$ on the carbon ink 
through a low-cost hydrothermal process. Glucose oxidase (GOx) is immobilized on the $\mathrm{ZnO} N W s$ by simple addition and drying. The paper channel layer and the WE layer are stacked together to form a complete E $\mu$ PAD with a three-electrode configuration. We characterize the device via a series of electrochemical measurements and demonstrate improved electrochemical responses of the device to the oxidation of glucose. The assay incubation time and the dose of pre-stored GOx are tuned to improve the sensing performance of the device. E $\mu$ PADs configured with varied WE areas and $\mathrm{ZnO}-\mathrm{NW}$ growth levels are calibrated; from these calibration data, a design for an E $\mu$ PAD that covers the range of clinically relevant glucose concentrations is selected for final calibration with spiked phosphate-buffered saline (PBS) and human serum. The reported ZnO-NW EMPAD has a linear response within the clinically relevant range for glucose sensing and provides a sensitivity and a limit of detection (LOD) that are both superior to existing E $\mu$ PADs and commercial meters.

\section{MATERIALS AND METHODS}

\section{Design and fabrication of ZnO-NW EuPADs}

An exploded schematic of the $\mathrm{ZnO}-\mathrm{NW}$ E $\mu \mathrm{PAD}$ is shown in Figure 1a. The device is assembled from two layers of chromatography paper (Whatman 1 CHR, GE Healthcare, Little Chalfont, Buckinghamshire, UK): (i) a top layer consisting of a wax-patterned hydrophilic reaction zone, a carbon counter electrode (CE) and a $\mathrm{Ag} / \mathrm{AgCl}$ reference electrode (RE) patterned on top of the reaction zone; and (ii) a bottom layer acting as a WE, which is made by stencil-printing carbon ink on paper and then hydrothermally growing $\mathrm{ZnO} \mathrm{NWs}$ on the carbon electrode. Instead of patterning all three electrodes on the same piece of paper (within the reaction zone), we chose to arrange the WE on a separate layer underneath the reaction zone. This design makes the hydrothermal growth of $\mathrm{ZnO}$ NWs on the carbon WE more straightforward. If the three electrodes were all patterned on top of the same reaction zone,
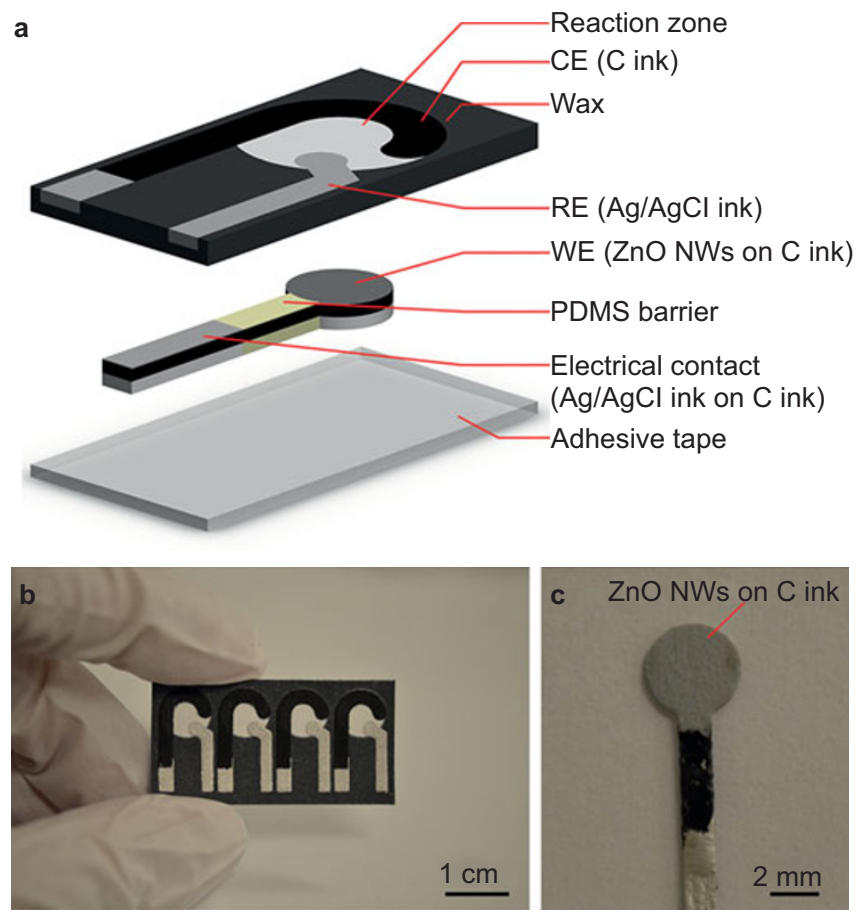

Figure 1 Design and fabrication of the ZnO-NW E $\mu$ PAD. (a) Schem-

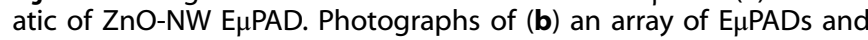
(c) a WE with ZnO NWs grown over its circular area (the gray color is from the $\mathrm{ZnO} N W s$ ). one would need to selectively grow $\mathrm{ZnO} \mathrm{NWs}$ on the WE only; this process requires the deposition of the ZnO-NP seeding solution onto the WE using techniques such as inkjet printing. This process is challenging and less consistent. Moreover, in our preliminary experiments, we found that the hydrothermal growth process compromised the hydrophobicity of the wax barrier of the paper reaction zone, making it impractical to selectively grow $\mathrm{ZnO}$ NWs on a WE patterned on top of a reaction zone. In addition, with a separate WE layer stacked underneath the reaction zone, we were able to achieve a large-sized WE without being limited by the sizes of the other two electrodes and the area of the reaction zone, and created an intimate contact between the ZnO NWs on the WE and the reaction zone; both the large WE and the close contact led to enhanced sensitivity in electrochemical detection with our $\mathrm{ZnO}$ NW E $\mu P A D$.

To fabricate the device, the reaction zone was patterned on the top layer via wax printing ${ }^{16,17}$. Solid wax was first printed on a piece of chromatography paper with a wax pinter (ColorQube 8570, Xerox, Wilsonville, OR, USA), and heated on a hot plate at $150{ }^{\circ} \mathrm{C}$ to form the hydrophilic paper reaction zones confined by hydrophobic wax barriers. A CE and an RE were then patterned on top of the reaction zone via stencil printing of carbon ink (E3456, Ercon, Wareham, MA, USA) and Ag/AgCl ink (E2414, Ercon, Wareham, MA, USA), respectively. A $\mathrm{Ag} / \mathrm{AgCl}$ strip was printed to connect the $C E$ and enhance the electrical conductivity between the $C E$ and the potentiostat. The inks were dried on a hot plate at $65{ }^{\circ} \mathrm{C}$ for $20 \mathrm{~min}$ after each step of the stencil printing. Another piece of chromatography paper was cut into the shape of the WE layer (Figure 1a) using a $\mathrm{CO}_{2}$ laser cutter (VLS 2.30, Universal Laser Systems, Scottsdale, AZ, USA), and carbon ink was printed on the circular side of the WE layer (Figure 2a). After the ink was dry, precured polydimethylsiloxane or PDMS ( $w / w$ ratio of base and curing agent: 10:1) was added to both surfaces of the CE layer to form thin films of hydrophobic barriers (Figure 1a) and confine liquids within the circular WE area during the $\mathrm{ZnO}-\mathrm{NW}$ growth and diagnostic assays. A $\mathrm{Ag} / \mathrm{AgCl}$ strip was patterned on the paper beam of the WE layer to create an electrical connection. The circular WE area was finally coated with ZnO NWs through a hydrothermal process, which will be described in detail in the Section of "Hydrothermal Growth of ZnO NWs on WE". Two sizes of circular WEs were fabricated and tested in this study with radii of $1.5 \mathrm{~mm}$ (WE \#1) and $2 \mathrm{~mm}$ (WE \#2).

Figure $1 \mathrm{~b}$ shows an array of four assembled ZnO-NW E $\mu$ PADs, which were fabricated simultaneously from the same process. The top paper layer with the four reaction zones and four sets of CEs and REs were conveniently attached to the bottom paper layer with four WEs using adhesive tape. The distance between the centers of the adjacent reaction zones was based on the standard tip-to-tip pitch $(9 \mathrm{~mm})$ of a multichannel pipette, so multiple reactions can be performed in parallel on such a sensor array by simultaneously adding sample solutions to different reaction zones with a multi-channel pipette. Figure 1c shows a photograph of the WE layer after growth of the ZnO-NWs. The circular area of the carbon ink turned from black to gray after the growth, indicating uniform coverage by the ZnO NWs.

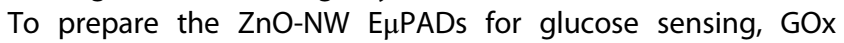
needs to be immobilized on the WE before assembly of the device. After a series of preliminary experiments, $5 \mu \mathrm{g}$ of $\mathrm{GOx}$ (Aspergillus niger, $147.9 \mathrm{U} \cdot \mathrm{mg}^{-1}$, Sigma-Aldrich, St. Louis, MO, USA) was chosen to be the enzyme dose added to each WE. GOx solutions $\left(1 \mu \mathrm{L}\right.$ of $5 \mathrm{mg} \mathrm{mL}^{-1}$ for WE \#1, and $2 \mu \mathrm{L}$ of $2.5 \mathrm{mg} \mathrm{mL}^{-1}$ for WE \#2) were dropped onto the circular area of the WE and dried at room temperature for $5 \mathrm{~min}$. A drop of $0.5 \%(\mathrm{w} / \mathrm{w})$ Nafion solution (1.3 $\mu \mathrm{L}$ for WE \#1, and $2 \mu \mathrm{L}$ for WE \#2) was then added onto the circular area and dried at room temperature for $5 \mathrm{~min}$. The Nafion coating over the WE stabilizes the GOx on the $\mathrm{ZnO} N W s^{11}$. 


\section{Hydrothermal growth of ZnO NWs on the WE}

The synthesis of $\mathrm{ZnO} N W s$ includes two steps: (i) seeding of $\mathrm{ZnO}$ nanoparticles (NPs) on WE and (ii) hydrothermal growth of ZnO NWs on the seeded $\mathrm{ZnO}-\mathrm{NPs}$. In the first step, $40 \mathrm{~mL}$ of $2 \mathrm{mM}$ zinc acetate dihydrate (ZAD) and $20 \mathrm{~mL}$ of $4 \mathrm{mM}$ sodium hydroxide (SH) solutions were prepared separately in pure ethanol, and the solutions were stirred on a hotplate at $70^{\circ} \mathrm{C}$ to completely dissolve the chemicals. The ZAD solution was heated in a $70{ }^{\circ} \mathrm{C}$ oven afterwards. After both the ZAD and $\mathrm{SH}$ solutions were cooled to $4^{\circ} \mathrm{C}$, the $\mathrm{SH}$ solution was slowly added to the ZAD solution with constant stirring, and the mixture was placed in a $60^{\circ} \mathrm{C}$ oven for $2 \mathrm{~h}$ to obtain a colloidal solution of ZnO NPs. The synthesized ZnO NPs have a uniform size of $3.06 \pm 0.58 \mathrm{~nm}(N=50)$.

The paper beams of the WE layer (coated with carbon ink and a PDMS barrier) were clapped between two $1 \mathrm{~mm}$ thick glass slides and the circular WE was exposed. The PDMS barriers on both sides of the paper beams constrained the subsequent seeding and hydrothermal growth solutions to the WE only, and no ZnO NWs grew on the paper beams (as confirmed via SEM imaging). A drop of ZnO-NP seeding solution ( $2.25 \mu \mathrm{L}$ for WE \#1, and $4 \mu \mathrm{L}$ for WE \#2) was then added to each circular WE. The carbon-ink electrode printed on paper is relatively hydrophobic but has an affinity to ethanol, and the seeding ethanol solution can thus spread well over the whole area of the circular WE. The WE layer was dried on a hot plate at $100^{\circ} \mathrm{C}$ for $3 \mathrm{~min}$. For each WE, this seeding and drying process was repeated 6 times to achieve a uniform coating of ZnO-NP seeds on the WE surface. After seeding, $25 \mathrm{~mL}$ of aqueous growth solution, which includes $50 \mathrm{mM}$ zinc nitrate hexahydrate, $25 \mathrm{mM}$ hexamethylenetetramine, and 0.372 $\mathrm{M}$ ammonium hydroxide, was prepared in a $25 \mathrm{~mL}$ glass bottle, and two arrays of four interconnected WEs were immersed in growth solution in the same bottle (Supplementary Figure S1). The glass bottle was then sealed and heated in an $86{ }^{\circ} \mathrm{C}$ oven for a certain period of time to allow the $\mathrm{ZnO}-\mathrm{NW}$ to grow. In principle, one could use as many bottles of growth solution as desired in a single run of ZnO-NW growth on a large number of WEs. The length and width of ZnO NWs grown on the WE can be controlled by adjusting the growth time $^{15}$; thus, one can tune the total surface area of the ZnO NWs on the WE and thus the device's sensitivity. After growth, the WE layer was thoroughly washed in deionized water and then in pure ethanol, and was dried before final device assembly.

\section{Electrochemical measurements}

For glucose detection, the three electrodes of the E $\mu$ PAD were connected by metal clips to a precision potentiostat (Autolab
PGSTAT302N, Metrohm, Mississauga, ON, Canada). $5 \mu \mathrm{L}$ of $1 \times$ PBS or human serum spiked with D-(+)-glucose (Sigma-Aldrich, St. Louis, MO, USA) was added to the reaction zone on the top of the sensor and wicked down to the WE. After incubation for $50 \mathrm{~s}$, the potentiostat started measurements at $100 \mathrm{~Hz}$.

\section{RESULTS}

Electron microscopy analysis of ZnO NWs grown on the WE We performed scanning electron microscopy (SEM) imaging of the WEs before and after hydrothermal growth. As shown in Figure 2a, the carbon WE before hydrothermal growth had a rough, plate-like surface morphology. After hydrothermal growth, a uniform layer of high-density ZnO NWs was formed on the entire WE (Figure 2b). Because of the rough surface of the carbon WE (Figure 2a), the synthesized $\mathrm{ZnO}$ NWs on the WE were not uniformly oriented in the same direction (Figure $2 \mathrm{~b}$ ). The average width of the obtained $\mathrm{ZnO} N W s$ was measured to be $298.72 \pm 54.11 \mathrm{~nm}(N=20)$. During SEM imaging, we conducted in-situ energy dispersive spectroscopy (EDS) analysis of the chemical composition of single ZnO NWs and observed clear peaks for zinc and oxygen in the obtained spectrum (Supplementary Figure S2), indicating that the NWs had the correct composition. Transmission electron microscopy (TEM) imaging of single $\mathrm{ZnO}$ NWs reveals lattice edges along the $\mathrm{ZnO}$ NW (Figure 2c). We measured the distance between two edges to be 0.262 $\mathrm{nm}$, which corresponds to the distance between adjacent (0002) planes in wurtzite $\mathrm{ZnO}$ crystal units.

\section{Characterization of ZnO-NW EuPADs}

To demonstrate the enhanced electrochemical performance of

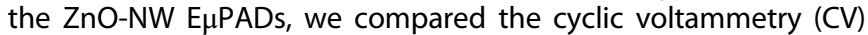

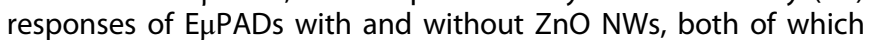
have the same WE dimensions (1.5 $\mathrm{mm}$ and $2 \mathrm{~mm}$ in radius) and GOx dose. We added $5 \mathrm{~mL}$ of pure PBS and $5 \mathrm{mM}$ glucose solution to the two types of devices and performed CV measurement after $50 \mathrm{~s}$ incubation. As shown in Figure 3, for both types

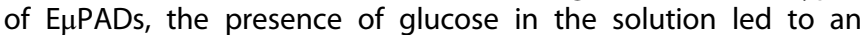
increase in the peak magnitude of measured CV current, which was caused by the GOx-catalyzed oxidation of glucose. In the non-NW E $\mu$ PADs, a clear increase in the CV current, in response to the presence of glucose, was observed at a CV potential of $\sim 0.8 \mathrm{~V}$ for both WE sizes. In contrast, the CV current response of the ZnO-NW E $\mu$ PADs started to increase at a CV potential as low as $0.3 \mathrm{~V}$ for both WE sizes. In addition, the peak values of the current
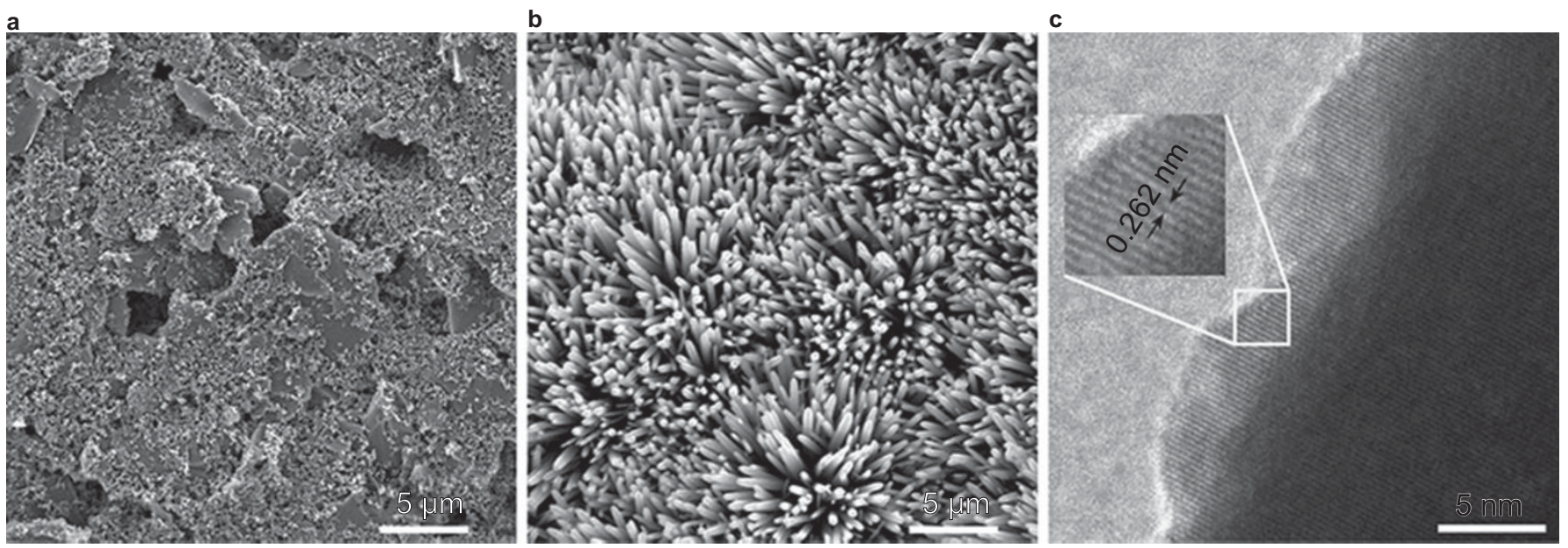

Figure 2 Growth and characterization of ZnO NWs. (a) SEM image of carbon-ink WE surface before seeding of ZnO NPs. (b) Zoomed-in view of ZnO NWs grown on carbon-ink WE. (c) TEM image of ZnO-NW crystal lattice. 

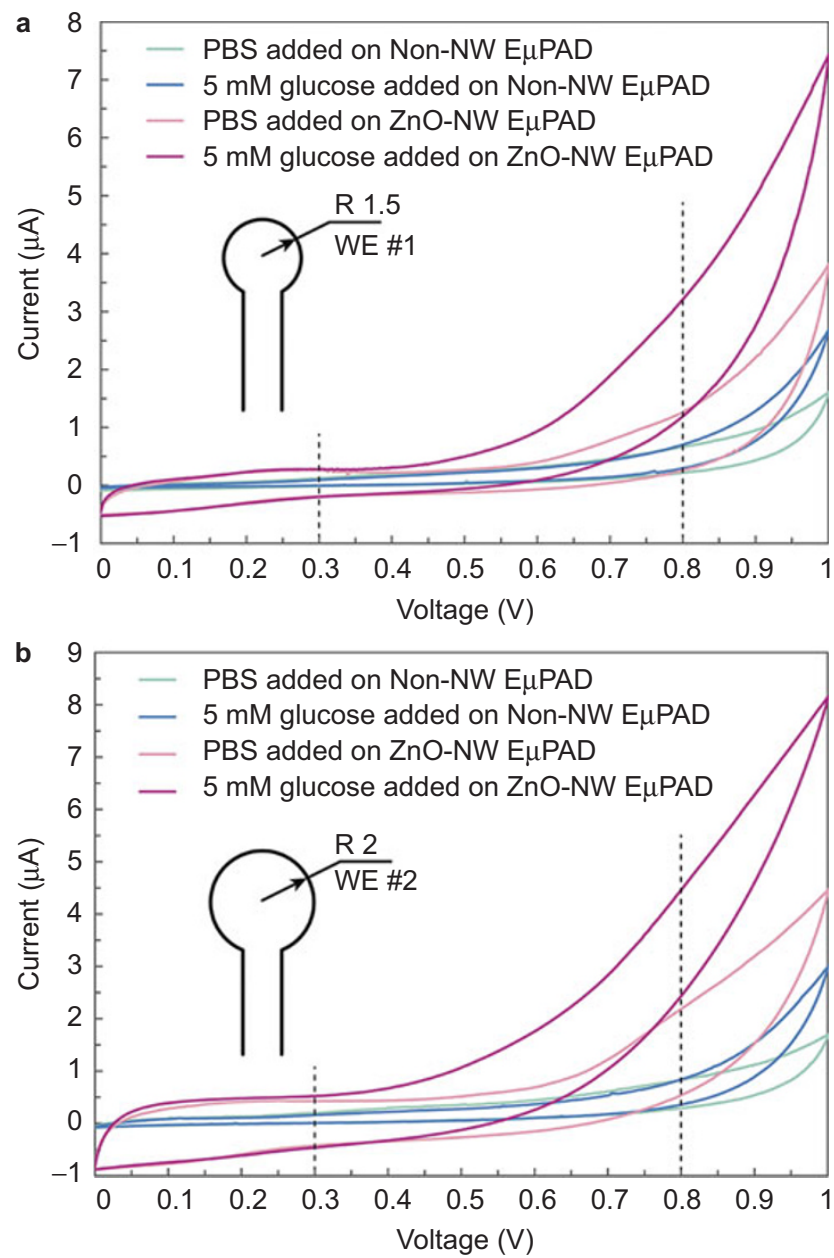

Figure $3 \mathrm{CV}$ characterization of $\mathrm{E} \mu \mathrm{PADs}$ with or without $\mathrm{ZnO}$ NWs. (a) CV responses of E $\mu$ PADs (with and without $\mathrm{ZnO} N W s$ ) using PBS or glucose, with WE \#1 (1.5 $\mathrm{mm}$ in radius). (b) CV responses of $\mathrm{E} \mu \mathrm{PADs}$ (with and without ZnO NWs) using PBS or glucose, with WE \#2 (2 $\mathrm{mm}$ in radius).

responses of the $\mathrm{ZnO}-\mathrm{NW} \mathrm{E} \mu \mathrm{PADs}$ were much higher than those of the non-NW E $\mu$ PADs (3.7 times higher for WE \#1, and 4.3 times higher for WE \#2).

Thus, we confirmed that ZnO NWs can improve the performance of glucose sensing, by both lowering the electrical potential required for the device to generate an electrochemical response and increasing the electrochemical current response. The ability to lower the potential for generating a current response is attributed to the low redox potential of $\mathrm{ZnO} N W s$, which is close to the oxidation potential of glucose $(0.124 \mathrm{mV} \text { or higher })^{11}$. The $\mathrm{ZnO} N W \mathrm{~s}$ on WE can increase the device's current response and thus its sensitivity for glucose detection primarily because they significantly increase the surface-to-volume ratio of the WE and thus enhance electron transfer at the electrode surface ${ }^{18}$. Moreover, the $\mathrm{ZnO}$ NWs are $n$-type semiconductor and has excellent electron mobility and surface charge transport efficiency, which also contributes to the enhanced current response of the ZnO-NW E $\mu$ PADs ${ }^{18}$. Additionally, in many other electrochemical glucose sensors (including paper-based sensors) that use GOx as the catalyst, electron mediators (typically $\mathrm{Fe}(\mathrm{CN})_{6}{ }^{3-} / \mathrm{Fe}(\mathrm{CN})_{6}{ }^{4-}$ ) are used to lower the working voltage and increase the current response ${ }^{5,19-}$ ${ }^{21}$. However, electron mediators are usually sensitive to light, and this sensitivity could cause biosensing instability. The integration of
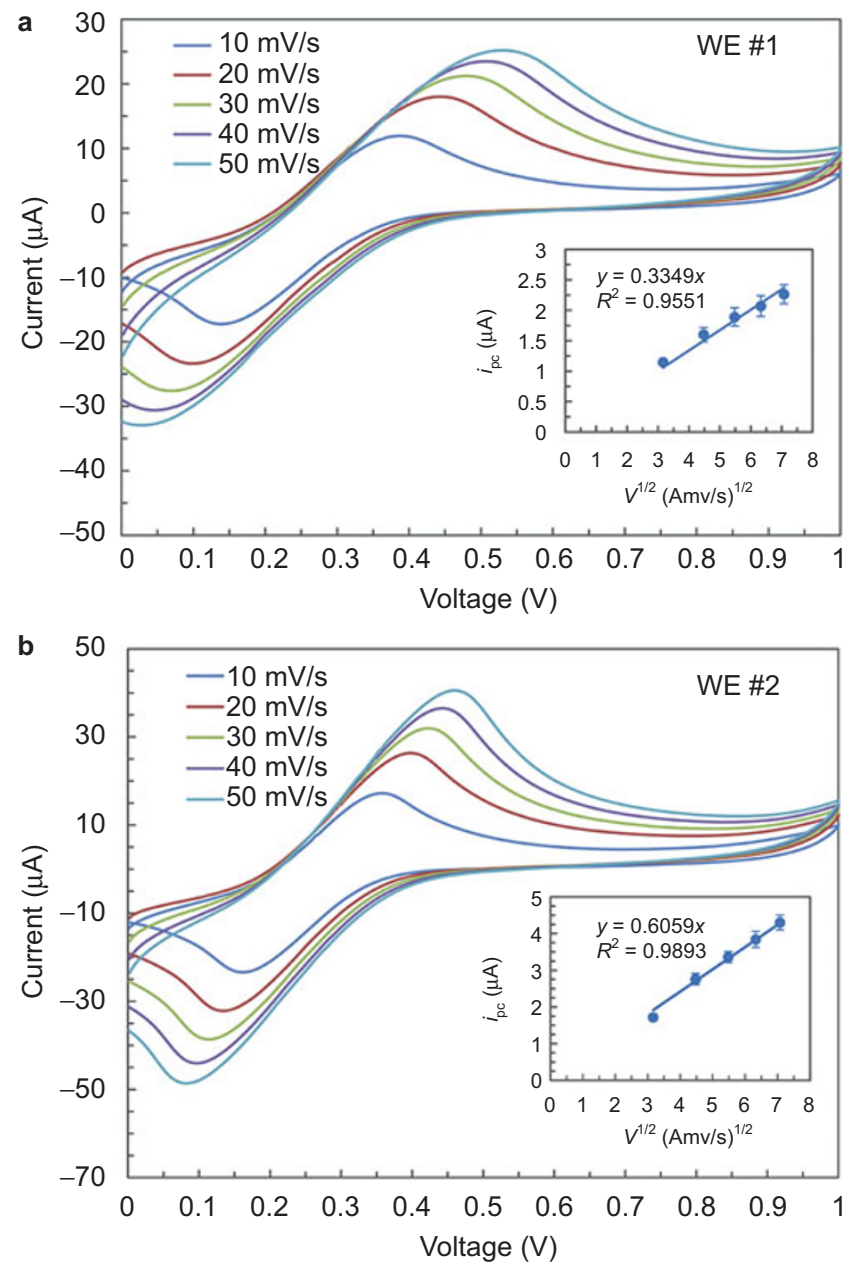

Figure $4 \mathrm{CV}$ characterization of $\mathrm{ZnO}-\mathrm{NW}$ E $\mu \mathrm{PADs}$ at varied scanning

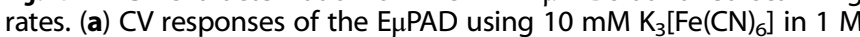
$\mathrm{KCl}$ aqueous solution at various scan rates, with WE \#1 $(1.5 \mathrm{~mm}$ in radius). (b) CV responses of the $\mathrm{E}_{\mu} \mathrm{PAD}$ using $10 \mathrm{mM} \mathrm{K}_{3}\left[\mathrm{Fe}(\mathrm{CN})_{6}\right]$ in $1 \mathrm{M}$ $\mathrm{KCl}$ aqueous solution at various scan rates, with WE \#2 (2 $\mathrm{mm}$ in radius). Insets: The linear relationship between the anodic peak current and the square root of the scan rate, measured from the CV $(N=5)$.

ZnO NWs into E $\mu$ PADs eliminates the need for electron mediators and thus can potentially improve the device stability.

We conducted CV characterization of the ZnO-NW E $\mu$ PADs using an aqueous solution of $10 \mathrm{mM} \mathrm{K}_{3}\left[\mathrm{Fe}(\mathrm{CN})_{6}\right]$ in $1 \mathrm{M} \mathrm{KCl}$. The $\mathrm{CV}$ response of the device shows typical redox peaks (Figure 4). The anodic current peak of the cyclic voltammogram was proportional to the square root of the CV scan rate for both WE sizes. These results indicate good electrochemical characteristics of the developed E $\mu$ PAD. However, we noticed that the distance between the redox peaks increased as the scan rate increased. This result is an indication of a slow electrochemical reaction, which is usually caused by limited mass transfer in an electrochemical cell and correspondingly, a thick diffusion layer. It is possible that the porous paper structure interferes with the mass transport in the paper reaction zone. Although the peak shift existed in the CV measurements and the shift level slightly varied from device to device, the device calibration results using chronoamperometry $(C A)$, to be presented later in the paper, reveal small device-to-device deviations. Therefore, the peak shift observed in multiple CV scans is less of a concern for one-time use of the device in glucose sensing. 


\section{Tuning the design parameters of the ZnO-NW E $\mu$ PAD}

The CV characterization results (Figures 3 and 4) imply the feasibility of operating the $\mathrm{ZnO}-\mathrm{NW}$ E $\mu$ PAD at voltage higher than $0.3 \mathrm{~V}$. In the following experiments, we chose $0.8 \mathrm{~V}$ to be the working voltage for $C A$ as a voltage of $0.8 \mathrm{~V}$ was widely used in previous $\mathrm{ZnO}-\mathrm{NW}$ glucose sensors ${ }^{11,22}$. As shown in typical CA curves (Figure 5a), the capacitive current quickly dropped after applying a $0.8 \mathrm{~V}$ voltage, and the Faradaic current eventually dominated the CA current response. After the current stabilized, we took the average of current values at $79-80 \mathrm{~s}$ to be the signal readout.

We conducted a series of experiments to select the proper design parameters for the device. First, we compared the preliminary CA calibration curves obtained with different incubation times (Supplementary Figure S3). At longer incubation times, the enzymatic detection of glucose generates a higher current, but the evaporation of the solution with time also decreases the current level. As shown in Supplementary Figure S3, an incubation time of $50 \mathrm{~s}$ generates a CA response which is similar to that measured at $70 \mathrm{~s}$ and higher than the responses at incubation times of $10 \mathrm{~s}$ and $30 \mathrm{~s}$. Therefore, we chose $50 \mathrm{~s}$ to be the incubation time for the final calibration experiments.
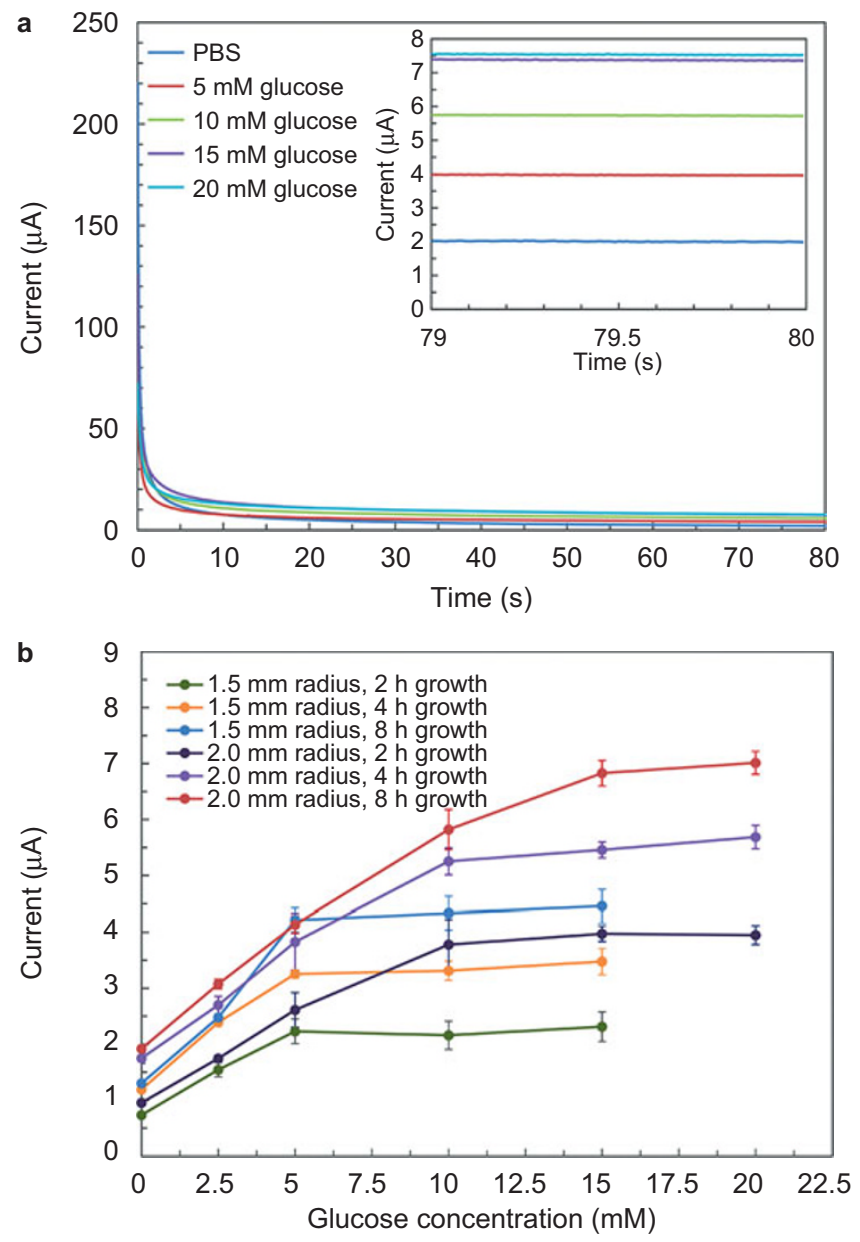

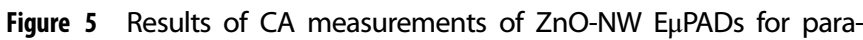
meter tuning. (a) Typical CA responses from ZnO-NW EMPADs with WE \#2 (2 mm in radius), ZnO NWs grown for $8 \mathrm{~h}$ and $5 \mu \mathrm{g}$ of GOx. (b) CA responses to different glucose concentrations which were obtained

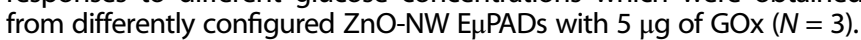

The second parameter we tuned is the dose of GOx added to the WE. We obtained preliminary CA calibration curves with GOx amounts between 1.25 and $20 \mu \mathrm{g}$. The results (Supplementary Figure S4) indicate that $5 \mu \mathrm{g}$ of GOx led to a high current response level and $10 \mu \mathrm{g}$ and $20 \mu \mathrm{g}$ of GOx did not generate appreciably higher current responses. This effect occurs probably because $5 \mu \mathrm{g}$ of GOx is already enough to cover the whole range of glucose

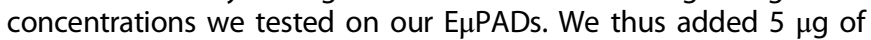
GOx to all of the E $\mu$ PADs used in the subsequent experiments.

The third parameter we investigated is the amount of $\mathrm{ZnO} N W s$ grown on the WE. It is well accepted that the length of $\mathrm{ZnO} N W s$ increases with growth time in the hydrothermal process ${ }^{23-25}$. In our study, it is difficult to measure the length of individual $\mathrm{ZnO}$ NWs because they are not uniformly aligned and their roots are hard to identify without sacrificing the WE layer. Instead, we measured the increases in masses of the WE pieces after hydrothermal growth for a certain period of time, which reflects the amount of $\mathrm{ZnO}$ NWs synthesized. The results are organized in Supplementary Figure S5. In consistency with previous observations $^{23-25}$, the mass of the synthesized ZnO NWs increased over the first $8 \mathrm{~h}$ of growth. Subsequently, further increases in the mass are marginal because of the depletion of chemicals in the growth solution. Thus, we grew $\mathrm{ZnO}$ NWs for no more than $8 \mathrm{~h}$ to prepare the devices for calibration. Another notable observation is that although WE \#1 has a smaller increase in mass than WE \#2 because of its smaller size (Supplementary Figure S5a), the increase in mass per area of WE \#1 is higher than that of WE \#2 after $4 \mathrm{~h}$ of growth (Supplementary Figure S5b). One possible explanation of this effect is that the larger area of WE \#2 consumed chemicals more effectively than the smaller area of WE \#1, making the ZnO-NW growth per area of WE \#2 slow down earlier than that of WE \#1.

In a more systematic series of experiments, we obtained the CA calibration curves for glucose detection with different combinations of WE sizes and ZnO-NW amounts, which are organized in

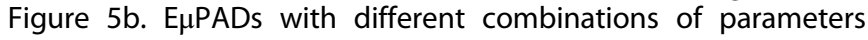
produced very different calibration curves in terms of sensitivity and linear range (Figure $5 \mathrm{~b}$ ). We recognized two major trends in the curves. First, a longer ZnO-NW growth time leads to higher sensitivity, primarily because longer ZnO NWs (corresponding to a larger amount of $\mathrm{ZnO} \mathrm{NWs}$ ) boost the current response to higher levels. Second, ZnO-NW EMPADs with WE \#1 have smaller linear ranges than devices with WE \#2 because the current responses become saturated more easily for the devices with smaller WE \#1 than for the ones with larger WE \#2, and this easier saturation leads to smaller linear ranges. Among the obtained calibration curves, devices with WE \#1 after growth of ZnO NWs for $8 \mathrm{~h}$ provide the highest sensitivity of $8.24 \mu \mathrm{A} \cdot \mathrm{mM}^{-1} \cdot \mathrm{cm}^{-2}$ (Figure $5 \mathrm{~b}$ ), with a corresponding LOD as low as $59.5 \mu \mathrm{M}$. However, its linear range is limited to $0-5 \mathrm{mM}\left(R^{2}=0.9877\right.$ in linear fitting). This systematic investigation illustrates the feasibility of finely tuning the LOD and sensitivity of our ZnO-NW E $\mu$ PADs by selecting the right combination of WE size and $\mathrm{ZnO}-\mathrm{NW}$ amount. We chose WE \#2 ( $2 \mathrm{~mm}$ in radius) and $8 \mathrm{~h}$ of $\mathrm{ZnO} \mathrm{NW}$ growth to fabricate the devices used for the final calibration experiments on serum samples. Devices with this configuration have a linear range up to $15 \mathrm{mM}$. Considering the medically relevant range of glucose concentrations (up to $3.89-6.67 \mathrm{mM})^{5}$, we believe this configuration covers the range related to medical applications.

\section{Calibration of ZnO-NW EuPADs for glucose detection}

Finally, we completed calibration experiments on both spiked PBS and human serum samples, using ZnO-NW E $\mu$ PADs with the previously determined parameters (the $2 \mathrm{~mm}$ radius WE \#2, $8 \mathrm{~h}$ of $\mathrm{ZnO} N W$ growth, addition of $5 \mu \mathrm{g}$ of GOx, and $50 \mathrm{~s}$ of incubation time). The calibration results from the spiked PBS reveal a linear range of $0-15 \mathrm{mM}$ (Figure $6 \mathrm{a}$ ), and the corresponding sensitivity 

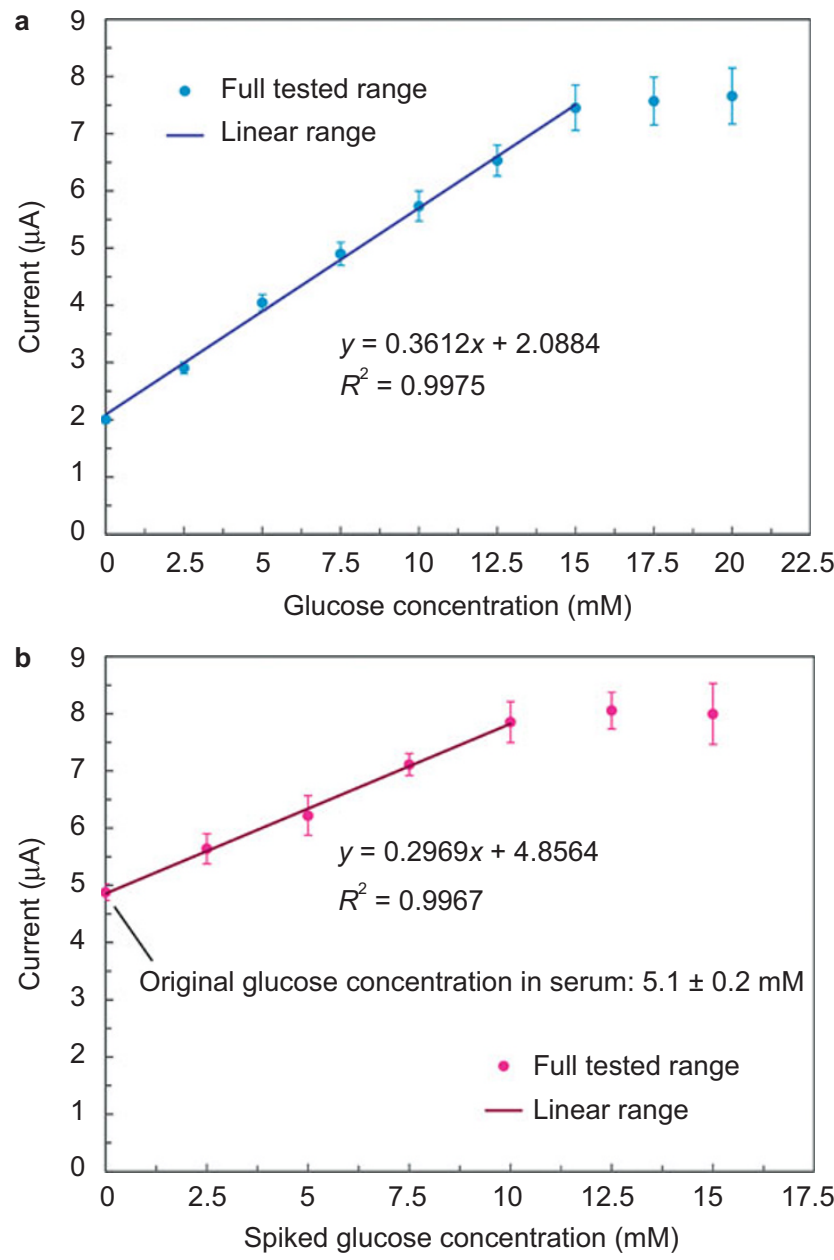

Figure 6 Calibration results for ZnO-NW $\mu$ PAD with WE \#2 (2 mm in radius), $8 \mathrm{~h}$ of $\mathrm{ZnO}$ NW growth and $5 \mu \mathrm{g}$ of GOx. (a) Calibration of ZnO-NW $\mu$ PAD in PBS $(N=5)$. (b) Calibration of $\mathrm{ZnO}-\mathrm{NW} \mu \mathrm{PAD}$ in human serum $(N=5)$.

and LOD are $2.88 \mu \mathrm{A} \cdot \mathrm{mM}^{-1} \cdot \mathrm{cm}^{-2}$ and $94.7 \mu \mathrm{M}$, respectively. The calibration experiments on spiked human serum show the performance of our devices for real glucose detection in a complex serum environment that contains various large molecules. To detect glucose in human serum, we re-performed the CV characterization to compare the responses of ZnO-NW E $\mu$ PAD and nonNW E $\mu$ PAD (Supplementary Figure S6), and confirmed that it is valid to use the same working voltage of $0.8 \mathrm{~V}$ in the CA-based calibration. The calibration results from the spiked serum samples revealed a linear range of $5.1-15.1 \mathrm{mM}$, and a sensitivity of 2.36 $\mu \mathrm{A} \cdot \mathrm{mM}^{-1} \cdot \mathrm{cm}^{-2}$ (Figure $6 \mathrm{~b}$ ). The initial concentration of $5.1 \mathrm{mM}$ on the serum calibration curve reflects the original glucose level of the un-spiked human serum, which was measured using a commercial glucose meter. The linear range of the $\mathrm{ZnO}-\mathrm{NW}$ E $\mu$ PADs covers the major clinically relevant range of glucose in human blood. We compared the performance of our devices with those of previously reported $\mathrm{E} \mu \mathrm{PADs}$ and commercial meters (all obtained with spiked PBS samples), and the sensitivities and LODs are summarized in Table 1. One can see that the sensitivities and LODs provided by our devices are superior to the commercial glucose meters and the previously reported $\mathrm{E} \mu \mathrm{PADs} \mathrm{s}^{3,5,19}$. Although we used human serum rather than whole blood in this work, it is totally feasible to integrate a layer of filtering paper/membrane on top of the device's reaction zone to filter out blood cells ${ }^{26}$. The above results
Table 1 Comparison of analytical performance of glucose sensors. Configuration \#1 has a working electrode with a radius of $1.5 \mathrm{~mm}$ and $\mathrm{ZnO} \mathrm{NWs}$ grown for $8 \mathrm{~h}$. Configuration \#2 has a working electrode with a radius of $2 \mathrm{~mm}$ and $\mathrm{ZnO} N W s$ grown for $8 \mathrm{~h}$

\begin{tabular}{|c|c|c|c|}
\hline Glucose sensor & Linear range $(\mathrm{mM})$ & $\begin{array}{l}\text { Sensitivity } \\
\left(\mu \mathrm{A} \cdot \mathrm{mM}^{-1} \cdot \mathrm{cm}^{-2}\right)\end{array}$ & $\operatorname{LOD}(\mu \mathrm{M})$ \\
\hline This work (configuration \#1) & $0-5$ & 8.24 & 59.5 \\
\hline This work (configuration \#2) & $0-15$ & 2.88 & 94.7 \\
\hline Previous $\mathrm{E}_{\mathrm{P}} \mathrm{PAD}^{19}$ & $0-20$ & 1.03 & 350.0 \\
\hline Previous $\mathrm{E} \mu \mathrm{PAD}{ }^{3}$ & $0-100$ & 4.92 & 210.0 \\
\hline Commercial meter ${ }^{5}$ & $0-30$ & $N / A$ & 833.0 \\
\hline
\end{tabular}

prove the effectiveness of our ZnO-NW E $\mu$ PADs for the detection of glucose in real blood samples.

We used $0.8 \mathrm{~V}$ as the working potential and obtained superior glucose sensing performance in spiked human serum. To further develop the ZnO-NW E $\mu$ PAD for practical applications, more experiments with patient samples need to be performed to examine any potential interference of other molecules with the electrochemical reaction used for glucose detection. We also need to study the stability of our device during long-term storage. For instance, ZnO NWs are sensitive to ultraviolet (UV) light, and their surface chemistry might be altered by long-term exposure to light. On the other hand, $\mathrm{ZnO} N W$ s become hydrophobic after stored in dark for long time, and become hydrophilic after exposed to UV irradiation ${ }^{27}$. Generally, hydrophilicity is desired for biosensing because the liquid samples can be better absorbed by a hydrophilic ZnO-NW electrode. Therefore, several optically induced phenomena might affect the biosensing performance of $\mathrm{ZnO}$ $\mathrm{NWs}$, and an effective storage method needs to be identified. The GOx also need to be stabilized through certain biochemical processes for room-temperature storage.

From the calibration results obtained with the spiked human serum, we were not able to quantify the LOD of our device. The human serum that we used has an initial glucose concentration of $5.1 \mathrm{mM}$ and thus does not allow us to measure the zeroconcentration background noise (i.e., the standard deviation of the readout signal from serum without glucose). Consequently, we were not able to accurately determine the LOD value, which should be the glucose concentration that generates an output signal 3 times the standard deviation of the signal from the zero-concentration serum. Nevertheless, one can notice that most of the standard deviation values of the output signals from human serum samples are similar in magnitude to those from PBS. Thus, using the standard deviation value of the signal from zero-concentration PBS, we estimated the LOD of our device for human serum to be $115.2 \mu \mathrm{M}$.

\section{DISCUSSION}

Based on the systematic experimental results, we validated the superior analytical performance of our ZnO-NW E $\mu$ PADs for glucose detection resulting from the hydrothermal growth of $\mathrm{ZnO} N W s$ on the WE. This improved performance relies on the following advantages of $\mathrm{ZnO}$ NWs for enzymatic sensing. (i) They have high surface-to-volume ratios, which significantly expand the surface of the electrode involved in the electrochemical reaction. Figure $5 b$ shows that the sensitivity increases with extended $\mathrm{ZnO}$-NW growth time, which leads to longer $\mathrm{ZnO} \mathrm{NWs}$ and thus a larger effective surface area on the WE. (ii) They immobilize enzymes more efficiently and stably due to their strong electrostatic attraction with the GOx. (iii) They are $n$-type semiconductor with a high carrier mobility and a high surface charge transfer efficiency ${ }^{28}$, which improves the device's electrochemical response to the GOxcatalyzed oxidation of glucose and eliminates the requirement for a light-sensitive electron mediator. (iv) From a practical point of 
view, the facile and low-cost hydrothermal growth process allows us to directly integrate $\mathrm{ZnO}$ NWs into the $\mu \mathrm{PAD}$ with high consistency, and no post hoc process is required.

We expect this novel means of direct $\mathrm{ZnO}-\mathrm{NW}$ synthesis on $\mathrm{E} \mu \mathrm{PAD}$ to play a powerful role in further explorations of using paper-based microfluidic platforms for highly sensitive electrochemical biosensing and point-of-care diagnoses. In addition to enzymatic glucose detection, $\mathrm{ZnO}$ NWs could also be surfacefunctionalized to covalently immobilize probe proteins ${ }^{29}$ or deoxyribonucleic acid (DNAs) ${ }^{30}$ and to perform antigen/antibody and DNA detection. The high sensitivity and high surface-tovolume ratio of $\mathrm{ZnO} \mathrm{NWs}$ promise improved biosensing performance of the E $\mu$ PADs. In addition, the ease and low cost of direct

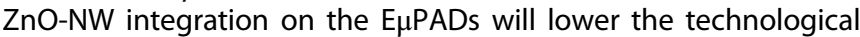
and economic barriers for popularizing these devices and will exert an even bigger impact on applications in the real world.

\section{CONCLUSION}

We developed a novel E $\mu$ PAD with ZnO NWs directly grown on the paper-based, carbon-ink WE, for high-sensitivity glucose detection. The synthesis of the $\mathrm{ZnO}$ NWs was completed through a low-cost, facile hydrothermal process. The integration of the $\mathrm{ZnO}$ NWs on the E $\mu$ PAD lowered the working voltage and boosted the device's current response in electrochemical detection, and the $\mathrm{ZnO}-\mathrm{NW}$ E $\mu$ PAD retained its proper characteristics as an electrochemical system. We conducted a series of experiments and determined the proper parameters of the ZnO-NW E $\mu$ PADs, such as the dose of GOx and the incubation time. More importantly, we compared different combinations of ZnO-NW growth levels and WE sizes, and demonstrated tunable analytical performance (sensitivity and LOD). Of the tested combinations of parameters, the $1.5 \mathrm{~mm}$ WE with $\mathrm{ZnO} \mathrm{NWs}$ grown for $8 \mathrm{~h}$ reached the highest sensitivity of $8.24 \mu A \cdot \mathrm{mM}^{-1} \cdot \mathrm{cm}^{-2}$ and a corresponding LOD of $59.5 \mu \mathrm{M}$. The E $\mu$ PADs with a $2 \mathrm{~mm}$ radius WE and $\mathrm{ZnO}$ NWs grown for $8 \mathrm{~h}$ were selected for detailed calibration using both spiked PBS and human serum and were demonstrated to have a linear range up to $15 \mathrm{mM}$ (covering the clinically relevant range of glucose concentrations in serum). The selected $\mathrm{ZnO}-\mathrm{NW}$ E $\mu$ PAD provided a sensitivity of $2.88 \mu \mathrm{A} \cdot \mathrm{mM}^{-1} \cdot \mathrm{cm}^{-2}$ and a LOD of $94.7 \mu \mathrm{M}$ in PBS. These results showed tunable biosensing performance that is superior to that of commercial glucose meters and previously reported E $\mu$ PADs.

\section{ACKNOWLEDGEMENTS}

This research was supported by the Natural Sciences and Engineering Research Council of Canada (NSERC) (Grant No. RGPIN 418553-12), the Canada Foundation for Innovation (Grant No. CFI-LOF 30316), and McGill University (Grant No. 120536). The authors also acknowledge financial support from the Canadian Research Chairs Program (Grant No. 237293), the McGill Chwang-Seto Faculty Scholarship Program (Grant No. 234304) to Xinyu Liu, and the NSERC-CREATE Training Program in Integrated Sensor Systems to Xiao Li and Chen Zhao.

\section{COMPETING INTERESTS}

The authors declare no conflict of interest.

\section{REFERENCES}

1 Martinez AW, Phillips ST, Whitesides GM. Three-dimensional microfluidic devices fabricated in layered paper and tape. Proceedings of the National Academy of Sciences 2008; 105: 19606-19611.

2 Martinez AW, Phillips ST, Whitesides GM et al. Diagnostics for the developing world: Microfluidic paper-based analytical devices. Analytical Chemistry 2010; 82: 3-10.

3 Dungchai W, Chailapakul O, Henry CS. Electrochemical detection for paperbased microfluidics. Analytical Chemistry 2009; 81: 5821-5826.

4 Zang DJ, Ge L, Yan M et al. Electrochemical immunoassay on a 3D microfluidic paper-based device. Chemical Communications 2012; 48: 4683-4685.
5 Nie ZH, Deiss F, Liu XY et al. Integration of paper-based microfluidic devices with commercial electrochemical readers. Lab on a Chip 2010; 10: 3163-3169.

6 Yetisen AK, Akram MS, Lowe CR. Paper-based microfluidic point-of-care diagnostic devices. Lab on a Chip 2013; 13: 2210-2251.

7 Patolsky F, Zheng G, Lieber CM. Nanowire sensors for medicine and the life sciences. Nanomedicine 2006; 1: 51-65.

$8 \mathrm{Wu}$ YF, Xue P, Kang YJ et al. Paper-based microfluidic electrochemical immunodevice integrated with nanobioprobes onto graphene film for ultrasensitive multiplexed detection of cancer biomarkers. Analytical Chemistry 2013; 85: $8661-8668$

9 Abbas A, Brimer A, Slocik JM et al. Multifunctional analytical platform on a paper strip: Separation, preconcentration, and subattomolar detection. Analytical Chemistry 2013; 85: 3977-3983.

10 Zhao ZW, Lei W, Zhang XB et al. ZnO-based amperometric enzyme biosensors. Sensors 2010; 10: 1216-1231.

11 Zang JF, Li CM, Cui XQ et al. Tailoring zinc oxide nanowires for high performance amperometric glucose sensor. Electroanalysis 2007; 19: 1008-1014.

12 Zhang FF, Wang XL, Ai SY et al. Immobilization of uricase on $\mathrm{ZnO}$ nanorods for a reagentless uric acid biosensor. Analytica Chimica Acta 2004; 519: 155-160.

$13 \mathrm{Gu} B X, \mathrm{Xu}$ CX, Zhu GP et al. Tyrosinase immobilization on $\mathrm{ZnO}$ nanorods for phenol detection. The Journal of Physical Chemistry B 2009; 113: 377-381.

$14 \mathrm{Ko} \mathrm{SH}$, Lee $\mathrm{D}$, Hotz $\mathrm{N}$ et al. Digital selective growth of $\mathrm{ZnO}$ nanowire arrays from inkjet-printed nanoparticle seeds on a flexible substrate. Langmuir 2012; 28: 4787-4792.

$15 \mathrm{Li} \mathrm{X}$, Wang $\mathrm{YH}$, Zhao $\mathrm{C}$ et al. Paper-based piezoelectric touch pads with hydrothermally grown zinc oxide nanowires. ACS Applied Materials \& Interfaces 2014; 6: 22004-22012.

$16 \mathrm{Li} \mathrm{X,} \mathrm{Liu} \mathrm{XY.} \mathrm{Fabrication} \mathrm{of} \mathrm{three-dimensional} \mathrm{microfluidic} \mathrm{channels} \mathrm{in} \mathrm{a} \mathrm{single}$ layer of cellulose paper. Microfluidics and Nanofluidics 2014; 16: 819-827.

17 Carrilho E, Martinez AW, Whitesides GM. Understanding Wax Printing: A simple micropatterning process for paper-based microfluidics. Analytical Chemistry 2009; 81: 7091-7095.

18 Wang JX, Sun XW, Wei A et al. Zinc oxide nanocomb biosensor for glucose detection. Applied Physics Letters 2006; 88: 233106-233108.

19 Zhao C, Thuo MM, Liu XY. A microfluidic paper-based electrochemical biosensor array for multiplexed detection of metabolic biomarkers. Science and Technology of Advanced Materials 2013; 14: 054402-054408.

20 Nie ZH, Nijhuis CA, Gong JL et al. Electrochemical sensing in paper-based microfluidic devices. Lab on a Chip 2010; 10: 477-483.

21 Wang J. Electrochemical glucose biosensors. Chemical Reviews 2008; 108: 814-825.

22 Pradhan D, Niroui F, Leung KT. High-performance, flexible enzymatic glucose biosensor based on $\mathrm{ZnO}$ nanowires supported on a gold-coated polyester substrate. ACS Applied Materials \& Interfaces 2010; 2: 2409-2412.

$23 \mathrm{Xu}$ CK, Shin P, Cao LL et al. Preferential growth of long ZnO nanowire array and its application in dye-sensitized solar cells. The Journal of Physical Chemistry $C$ 2010; 114: 125-129.

24 Qiu JJ, Li XM, Zhuge FW et al. Solution-derived $40 \mu \mathrm{m}$ vertically aligned $\mathrm{ZnO}$ nanowire arrays as photoelectrodes in dye-sensitized solar cells. Nanotechnology 2010; 21: 195602-195610.

25 Law M, Greene LE, Johnson JC et al. Nanowire dye-sensitized solar cells. Nature Materials 2005; 4: 455-459.

26 Songjaroen T, Dungchai W, Chailapakul O et al. Blood separation on microfluidic paper-based analytical devices. Lab on a Chip 2012; 12: 3392-3398.

27 Feng XJ, Feng L, Jin $\mathrm{MH}$ et al. Reversible super-hydrophobicity to superhydrophilicity transition of aligned $\mathrm{ZnO}$ nanorod films. Journal of the American Chemical Society 2004; 126: 62-63.

$28 \mathrm{Xu} \mathrm{S}$, Wang ZL. One-dimensional $\mathrm{ZnO}$ nanostructures: solution growth and functional properties. Nano Research 2011; 4: 1013-1098.

$29 \mathrm{Yu}$ RM, Pan CF, Wang ZL. High performance of ZnO nanowire protein sensors enhanced by the piezotronic effect. Energy \& Environmental Science 2013; 6: 494-499.

30 Niepelt R, Schroder UC, Sommerfeld J et al. Biofunctionalization of zinc oxide nanowires for DNA sensory applications. Nanoscale Research Letters 2011; 6: 511-517.

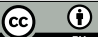

This work is licensed under a Creative Commons Attribution 4.0 Unported License. The images or other third party material in this article are included in the article's Creative Commons license, unless indicated otherwise in the credit line; if the material is not included under the Creative Commons license, users will need to obtain permission from the license holder to reproduce the material. To view a copy of this license, visit http://creativecommons. org/licenses/by/4.0/ 